\title{
Comparative studies of dual bronchodilation in COPD
}

\author{
Mario Cazzola $^{1}$, Maria Gabriella Matera ${ }^{2}$, Paola Rogliani ${ }^{1}$, Luigino Calzetta ${ }^{3}$ \\ ${ }^{1}$ Unit of Respiratory Medicine, Department of Experimental Medicine, University of Rome "Tor Vergata", Rome; \\ ${ }^{2}$ Unit of Pharmacology, Department of Experimental Medicine, University of Campania "Luigi Vanvitelli", Naples; \\ ${ }^{3}$ Unit of Respiratory Disease and Lung Function, Department of Medicine and Surgery, University of Parma, Italy
}

\begin{abstract}
Dual bronchodilation therapy is becoming the cornerstone for the treatment of COPD because the clinical benefits of
\end{abstract}

Correspondence: Prof. Mario Cazzola, Unit of Respiratory Medicine, Department of Experimental Medicine, University of Rome "Tor Vergata", Roma, Italy.

E-mail: mario.cazzola@uniroma2.it

Conference presentation: This paper was first presented as a keynote lecture at the $11^{\text {th }}$ Virtual and Interactive Congresso de Pneumologia do Centro-Ibérico, 1 and 2 October 2020.

Key words: Dual bronchodilators; COPD; comparative effectiveness studies.

Author contributions: $\mathrm{MC}$ was involved in the conception of the idea, literature review, drafting the manuscript, editing and finalization of the manuscript; MGM, PR, LC were involved in conception of the idea, literature review, critical review, editing and finalization of the manuscript. All the authors gave their approval to the final submitted version of the manuscript.

Conflict of interest: MC reports grants and personal fees from Almirall, Boehringer Ingelheim, Novartis, and Zambon and personal fees from ABC Farmaceutici, AstraZeneca, Biofutura, Chiesi Farmaceutici, Cipla, Edmond Pharma, GlaxoSmithKline, Lallemand, Menarini, Mundipharma, Ockham Biotech, Pfizer, Sanofi, and Verona Pharma, outside the submitted work. MGM reports grants and personal fees from GlaxoSmithKline, and Novartis and personal fees from ABC Farmaceutici, AstraZeneca, and Chiesi Farmaceutici, outside the submitted work. PR reports grants and personal fees from Almirall, Boehringer Ingelheim, Chiesi Farmaceutici, Novartis and Zambon, and personal fees from AstraZeneca, Biofutura, Edmond Pharma, GlaxoSmithKline, and Sanofi, outside the submitted work. LC reports grants and personal fees from Boehringer Ingelheim, and Novartis, grants from Chiesi Farmaceutici, personal fees from ABC Farmaceutici, Edmond Pharma, Ockham Biotech, Verona Pharma and Zambon, grants from Almirall, and non-financial support from AstraZeneca, outside the submitted work.

Received for publication: 26 September 2020.

Accepted for publication: 11 December 2020.

${ }^{\circ}$ Copyright: the Author(s), 2021

Licensee PAGEPress, Italy

Monaldi Archives for Chest Disease 2021; 91:1625

doi: 10.4081/monaldi.2021.1625

This article is distributed under the terms of the Creative Commons Attribution Noncommercial License (by-nc 4.0) which permits any noncommercial use, distribution, and reproduction in any medium, provided the original author(s) and source are credited.
LABA/LAMA fixed-dose combinations (FDCs) are now extensively established. Therefore, it not surprising that a number of LAMA/LABA combinations in a single inhaler have now been approved for clinical use as treatments for patients with COPD. Regrettably, very few head-to-head studies between all of the available LABA/LAMA FDCs have been carried out. This makes choosing the most appropriate FDC difficult. Comparative effectiveness research that also uses conventional meta-analyses to compare different care strategies can help generate useful information. A bidimensional comparative analysis across LAMA/LABA FDCs has suggested constant superiority for tiotropium/olodaterol. However, considering that there is not an equivalent amount of evidence on efficacy outcomes for all LAMA/LABA FDCs, a proper comparison between the different LAMA/LABA FDCs cannot be made yet, and the information available is still rather inconsistent.

\section{Importance of dual bronchodilation}

According to GOLD strategy document, long-acting bronchodilator monotherapy is recommended for low-grade persistent symptoms, for patients with a high symptom burden but not frequent or severe exacerbations and for frequent exacerbators with lower symptom burden, with escalation to dual bronchodilator therapy for persistent symptoms [1]. However, for patients with severe dyspnea, initial therapy with two bronchodilators is also a possibility to be considered. Long-acting muscarinic antagonist (LAMA)/long-acting $\beta_{2}$-agonist (LABA) combinations are also recommended for those patients with recurrent exacerbations on LAMA monotherapy [1].

We have many doubts about the validity of starting the treatment with long-acting bronchodilator monotherapy [2]. Actually, there is broad evidence that in everyday (real-life) conditions, many patients receiving long-acting bronchodilator monotherapy continue to experience significant symptoms at any level of COPD severity [3]. Therefore, a more aggressive therapy may be indicated. This is possible by increasing the dose of the bronchodilator with the evident higher risk of adverse events related to the class of bronchodilators used. $\beta_{2}$-Agonist stimulate the heart inducing increased heart rate and palpitations, because some of the adrenoceptors (ARs) in the atria and ventricles are $\beta_{2}$-ARs, produce metabolic actions such as glycogenolysis in liver and skeletal muscle and also endocrine actions, increasing insulin and glucagon release [4-6]. Dry mouth, narrow-angle glaucoma, and urinary retention in older men with prostatic hyperplasia are common adverse events with muscarinic antagonists $[4,6,7]$. Concerns have been raised about the possible association of the use of these antagonists with cardiovascular morbidity (tachycardia and arrhythmia) and mortality [8]. However, it seems that LAMAs do not increase the risk of cardio- 
vascular severe adverse events compared to other active therapies or placebo regardless of the device, even in patients with coexisting cardiovascular diseases [8]. In any case, an additional dose does not induce any substantial improvement in lung function in many patients [9]. Therefore, an alternative therapeutic option is to add a second bronchodilator with a different mechanism of action [10].

The results of our systematic review with meta-analysis of dual bronchodilation with LAMA/LABA for the treatment of stable COPD that incorporated the data from all LAMA/LABA fixed dose combination (FDC) trials lasting at least 3 months, showed that all LAMA/LABA combinations are always more effective than the LAMA or LABA alone in terms of the improvement in trough forced expiratory volume in one second $\left(\mathrm{FEV}_{1}\right)$, dyspnea as assessed by the transitional dyspnea index (TDI) and health-related quality of life as assessed by the St. George's Respiratory Questionnaire (SGRQ) scores [11]. Furthermore, in another meta-analysis, we documented that if an increase in exercise capacity in patients with COPD is the goal of the therapy, LAMA/LABA combinations are more effective than their monocomponents in improving endurance time (ET) and inspiratory capacity (IC) [12].

Indeed, pharmacological studies strongly support such evidence. Several mechanisms have been suggested to explain the synergy between LAMAs and LABAs at the airway smooth muscle (ASM) level and presynaptically in parasympathetic nerve fibres [13]. Remarkably, there is a crosstalk induced by the activation of $\beta_{2}$-ARs and the inhibition of muscarinic $\mathrm{M}_{3}$ receptors at the level of ASM cell that leads to synergistic bronchorelaxant effect, with a greater relaxation of ASM than using the monocomponents [14].

Apart from this potential synergistic effect on ASM, LAMA/LABA combinations prevent or delay acute exacerbations of COPD (AECOPDs) when compared with LAMA monotherapy [15]. As already mentioned, for frequent exacerbators with lower symptom burden, GOLD strategy recommends using a LAMA as preferred monotherapy [1]. There are several mechanisms by which LAMA/LABA combinations may decrease the frequency of AECOPDs [16]. They decrease lung hyperinflation and symptom severity, improve sputum clearance by reducing mucus hypersecretion and increasing mucociliary clearance, and improve symptoms reducing their variability by "stabilizing" the airways in patients with COPD. They also elicit anti-inflammatory actions that have been demonstrated in vitro and in experimental models but have not been confirmed yet in patients with COPD [16].

We have demonstrated that co-administration of LAMAs and LABAs also leads to a reduction in the release of non-neuronal acetylcholine (ACh) that is released locally in response to inflammatory stimuli from epithelial cells lining the airways known to contain choline acetyltransferase activity [17]. Non-neuronal ACh has inflammatory properties mainly via muscarinic $\mathrm{M}_{3}$ receptors [18]. The multitude of cells in the airways, including bronchial epithelial cells, neutrophils, lymphocytes, macrophages and fibroblasts, has muscarinic receptors. LAMAs and LABAs synergistically inhibit the release of non-neuronal ACh from bronchial epithelial cells through the modulation of organic cation transporter 1, a transporter for the release of ACh from airway epithelial cells [18]. The capacity of LABA and LAMA to synergistically inhibit the release of proinflammatory factors from parenchymal cells or inflammatory cells is another possible mechanism [18].

All our research on dual bronchodilation leads us to believe that this therapeutic approach is extremely useful in COPD [19]. Indeed the synergistic relaxant effect of LAMA/LABA combinations at the level of medium bronchi documented in several translational studies $[20,21]$ is related with an improvement in $\mathrm{FEV}_{1}$. The synergistic interaction on small airways [17,22-24] explains the reduction of lung hyperinflation that decreases dyspnea and other symptoms and improves exercise tolerance. The impact on the non-neuronal cholinergic system [17] may explain the protective effect against COPD exacerbations.

We firmly believe that it is useful to start treating COPD with dual bronchodilation from the time of the first diagnosis, in order to optimize bronchodilation while interfering with the pathways that influence airway tone [25]. At present, we must administer the currently approved doses for treating COPD, but we are confident that the documented pharmacological synergism of action between LAMA and LABA should lead to verifying whether lower dosages can be equally effective. If this approach will be positive, we will certainly succeed in reducing the risk of adverse events that characterize both LAMAs and LABAs when they are taken at the full doses currently approved for the treatment of COPD, while satisfying the need to optimize bronchodilation. It remains to be seen whether any supposed synergy would allow a dose reduction of both component drugs whilst still inducing clinical meaningful bronchodilation over a $24 \mathrm{~h}$ period.

\section{How to choose a LAMA/LABA combination}

For all these features, it not surprising that a number of LAMA/LABA combinations in a single inhaler have now been approved for clinical use as treatments for patients with COPD [4]. However, it must be pointed out that there are currently no recommendations that make any specific LAMA/LABA combination preferable over another and, in any case, no LAMA/LABA therapy is approved for specific subgroups of patients with COPD [4]. Therefore, we are far from being able to apply a personalized approach when using dual bronchodilation in COPD. At present time, the choice of LAMA/LABA should be individualized for each patient's preferences and degree of disease burden.

In any case, there is a fundamental question that should be answered: "Which of these combinations, when translated into practice, improve care and increase the likelihood of health benefits?" [26]. Unfortunately, there is little information to answer this question. There are two studies that have compared two LAMA/LABA combinations in a head-to-head comparison. In the first study glycopyrronium/indacaterol and umeclidinium/vilanterol provided clinically meaningful and comparable bronchodilation in patients with moderate-to-severe COPD [27]. In the second trial, superiority was observed for the primary end point of trough $\mathrm{FEV}_{1}$ at week 8 with umeclidinium/vilanterol compared with tiotropium/olodaterol in patients with symptomatic COPD [28].

We fully share the view that the outcome of a trial that shows the superiority of one treatment over another cannot be extrapolated to a larger and less selected population, even if it is obtained using a large number of patients [29]. Large head-to-head equivalence trials comparing LAMA/LABA FDCs are crucial to establish the real superiority of one combination over the others.

In our opinion, prescribers should always consider the pharmacological profile of the LAMA/LABA combination they would like to recommend. In fact, fundamental pharmacological characteristics, such as intrinsic activity, dissociation rate and residence half-life of the LAMA and LABA included in each LAMA/LABA combination differ from those of the other combinations [14]. These substantial differences should guide them in choosing the combination to use.

Another very important aspect to consider is the pharmacokinetic/pharmacodynamic interaction related to the concentrations of the drugs included in the combination. It is well known that the optimal 
condition to induce pharmacological synergy is to administer the drugs at isoeffective concentrations [30]. A specific range of concentration-ratio at which the drug mixture may induce appreciable synergistic interaction characterizes each LAMA/LABA combination [31]. In some of the currently marketed LAMA/LABA FDCs the monocomponents were not adequately balanced, with the bronchorelaxant effect mainly due to the action of the LAMA [31]. This probably prevented us from detecting any synergistic interaction not only in ex vivo studies but also in randomized controlled trials (RCTs) [31].

Considering the available dual bronchodilator combinations, adding a LABA to a LAMA in patients with COPD induces a further overall clinically relevant increase in $\mathrm{FEV}_{1}$ of $\approx 65 \mathrm{~mL}$, when the drug mixtures are administered at the currently approved doses [31]. Nevertheless, and remarkably, when olodaterol $10 \mu \mathrm{g}$ is added to tiotropium $5 \mu \mathrm{g}$ the improvement in $\mathrm{FEV}_{1}$ even doubles, regardless of any synergistic interaction proved for this combination in COPD patients [31].

A systematic review without meta-analysis conducted two years ago critically examined the available evidence on marketed LABA/LAMA FDCs or those under development in terms of clinical relevance for the management of COPD. It allowed us to note that evidence supporting the efficacy of LAMA/LABA FDCs for COPD is heterogeneous, particularly for TDI and SGRQ scores, exacerbation rates, ET, and IC [32]. All this led us to conclude that the published evidence did not allow to establish the equivalence of all LABA/LAMA FDCs.

However, the comparative effectiveness research addresses possible harms and benefits for heterogeneous patient populations in heterogeneous healthcare settings also using conventional metaanalyses to compare different care strategies [26]. Consequently, we have performed a bidimensional comparative analysis across LAMA/LABA FDCs [33]. Data resulting from the network metaanalysis across LAMA/LABA FDCs indicated that tiotropium/olodaterol $5 / 5$ was significantly more effective than both aclidinium/formoterol 400/12 and glycopyrronium/formoterol 14.4/9.6 in improving trough $\mathrm{FEV}_{1}$, and significantly more effective than glycopyrronium/formoterol 14.4/9.6 in improving TDI. Also umeclidinium/vilanterol 62.5/25 significantly improved $\mathrm{FEV}_{1}$ when compared to glycopyrronium/formoterol 14.4/9.6. The risk of cardiovascular severe adverse events was significantly greater in patients treated with glycopyrronium/indacaterol 50/110 than in those treated with aclidinium/formoterol 400/12. The surface under the cumulative ranking curve (SUCRA) indicated a specific rank of effectiveness with respect to the improvement in trough $\mathrm{FEV}_{1}$, TDI and SGRQ. Results showed constant superiority for tiotropium/olodaterol. Concerning the cardiovascular safety profile, aclidinium/formoterol was better than tiotropium/olodaterol, which in any case appeared superior to all the other FDCs also for this aspect. We also calculated the novel Improved Bidimensional SUCRA (IBiS) score to define the combined efficacy/safety profile of LAMA/LABA FDCs. The ranking provided by this score allows identifying what is the best LABA/LAMA FDC in agreement with the multifaceted functional, clinical, and cardiovascular safety needs of each COPD patient [34]. Also the evaluation of this score highlighted the superiority of tiotropium/olodaterol [33].

\section{Tiotropium/olodaterol FDC}

The consequence of these indirect clinical findings was the need to characterize the pharmacological interaction between tiotropium and olodaterol on the human airways. We documented that these two bronchodilators induced a synergistic relaxant response in both medium isolated bronchi and small airways submaximally pre-contracted by carbachol, which was significant at low concentrations [35]. Combining tiotropium with olodaterol induced a $22 \%$ increase in the releasing response of medium bronchi and a $26 \%$ increase in that of small airways compared to the expected additive response calculated via the Bliss Independence test. The Bliss Independence is one of the most commonly used models to study combined effects of substances in vivo, ex vivo and in vitro [36]. Also the Unified Theory analysis was carried out, in order to adequately quantify the magnitude of synergism through the Combination Index outcome. This analysis confirmed that tiotropium/olodaterol combination elicited a strong-tovery strong synergistic interaction in both medium bronchi and small airways through a wide range concentration. The extent of bronchial relaxation in response to transmural stimulation was synergistically greater when tiotropium and olodaterol were administered in combination compared with the effect elicited by the monocomponents. Importantly, the duration of the synergistic interaction between tiotropium and olodaterol was markedly long and detectable for about $9 \mathrm{~h}$, after which an additive interaction was recorded.

Co-administration of tiotropium and olodaterol, but also tiotropium bromide and olodaterol, administered alone, reduced the release of non-neuronal ACh from isolated bronchi, whereas olodaterol but not tiotropium bromide enhanced cAMP levels and the combination induced a further increase [35]. This pharmacological characterization suggests that tiotropium/olodaterol combination has a pharmacodynamic profile at least similar to that of glycopyrronium/indacaterol, which in the Effect of QVA149 Versus NVA237 and Tiotropium on Chronic Obstructive Pulmonary Disorder Exacerbations (SPARK) study was superior in preventing moderate to severe AECOPDs compared with the single LAMA glycopyrronium but not with tiotropium [15]. Therefore, tiotropium might protect against AECOPDs with mechanisms other than bronchodilation [37].

In effect, the Tiotropium and olodaterol in the prevention of chronic obstructive pulmonary disease exacerbations (DYNAGITO) study, which evaluated tiotropium and olodaterol in the prevention of AECOPDs, documented that the tiotropium/olodaterol FDC did not influence the exacerbation rate compared with tiotropium alone [38]. As we wrote in the editorial that commented on this study, a main issue biased the study [39]. The majority of patients $(70 \%)$ were taking an inhaled corticosteroid (ICS) at baseline and did not stop this treatment. This means that a huge number of individuals in the tiotropium group actually received tiotropium plus ICS and a large body of evidence shows that ICSs reduce the risk of AECOPDs [40]. The results of this study suggested that tiotropium combined with an ICS is almost as effective as the tiotropium/olodaterol combination in lowering the AECOPD risk. Therefore, an ICS/LAMA combination might be considered an option for treating COPD in patients with a history of AECOPDs [39].

However, going back to what has already been described, we have shown that even tiotropium administered alone, reduced the release of non-neuronal ACh from isolated bronchi [35]. In addition, tiotropium is able to control the proinflammatory activity of interleukin (IL)-8 and IL-17A in human bronchial epithelial cell lines [41]. Furthermore, it has also been shown that tiotropium alone, regardless if combined with olodaterol, reduces transforming growth factor- $\beta_{1}$-mediated neutrophilic inflammation in COPD [42]. These effects alone may explain the ability of tiotropium to reduce the risk of AECOPDs.

Nevertheless, in the DYNAGITO study, the difference in the reduction the rate of exacerbations did not achieve statistical significance, possibly as a result of the statistical method used. A pooled analysis of data from almost 10,000 patients with moder- 
ate-to-very severe COPD from three 1-year studies demonstrated that dual bronchodilation with tiotropium/olodaterol reduced moderate/severe exacerbations and exacerbations leading to hospitalisation compared with tiotropium in a range of patient subgroups, including GOLD stage 2-3 patients, in patients with varying exacerbation histories, and in patients receiving an ICS at baseline [43].

Considering that it has been suggested that tiotropium Respimat significantly increases the risk of mortality and tiotropium/olodaterol FDC is delivered via the Respimat Soft Mist Inhaler [44], we have also carried out a meta-analysis aimed to assess whether the favorable efficacy profile of tiotropium/olodaterol is complemented by a safe cardiovascular profile in COPD patients enrolled in RCTs [45]. Tiotropium/olodaterol was characterized by a satisfactory cardiovascular safety profile vs monocomponents, with no effect on the risk of arrhythmia, myocardial infarction, stroke, and mortality, and limited not significant impact on the frequency of heart failure in COPD patients.

This is reassuring information, but it should nevertheless be stressed that it has been shown that there is no risk in treating patients with cardiovascular disease (a history of myocardial infarction, cerebrovascular accidents, or cardiac arrhythmia) who are suffering from concomitant COPD with tiotropium/olodaterol, even when a $\beta$-AR blocker is added [46]. This finding is important because, although $\beta$-AR blockers may have beneficial effects in patients with COPD independent from cardiovascular effects [47], many physicians withhold $\beta$-AR blockers from patients with chronic airways disorders having concerns about bronchoconstriction and neutralization of the effectiveness of $\beta_{2}$-AR agonists [48]. We were invited to comment on this article [49] and fully agreed on the possibility of combining $\beta$-AR blockers and dual bronchodilators also because there is experimental evidence that the co-administration of a $\beta_{2}$-AR agonist and a $\beta_{1}$-AR blocker can influence cardiac remodeling [50]. However, we could not avoid pointing out some weaknesses in the study, especially the fact that in the $\beta$-AR blocker group patients had less severe COPD and a higher mean baseline postbronchodilator $\mathrm{FEV}_{1}$ and also took cardiovascular drugs that may have affected the results.

\section{Conclusions}

In conclusion, we strongly believe that each available LAMA/LABA FDC has a specific efficacy/safety profile that needs to be considered for personalized therapy in COPD. In any case, we fully agree that there is not an equivalent amount of evidence on efficacy outcomes for all LAMA/LABA FDCs. As a result, a proper comparison between the different LAMA/LABA FDCs cannot be made yet, and the information available is still rather inconsistent.

Only for glycopyrronium/indacaterol and tiotropium/olodaterol we have an appreciable amount of information on efficacy and safety thanks to the clinical development programs Ignite and Tovito. For this reason, care should be taken when extrapolating the findings for each LABA/LAMA FDC to the entire drug class [51].

\section{References}

1. Singh D, Agusti A, Anzueto A, et al. Global strategy for the diagnosis, management, and prevention of chronic obstructive lung disease: the GOLD science committee report 2019. Eur Respir J 2019;53:1900164.
2. Cazzola M, Rogliani P, Stolz D, Matera MG. Pharmacological treatment and current controversies in COPD. F1000Res 2019;8:F1000 Faculty Rev-1533.

3. Dransfield MT, Bailey W, Crater G, et al. Disease severity and symptoms among patients receiving monotherapy for COPD. Prim Care Respir J 2011;20:46-53.

4. Cazzola M, Page CP, Calzetta L, Matera MG. Pharmacology and therapeutics of bronchodilators. Pharmacol Rev 2012;64:450-504.

5. Cazzola M, Page CP, Rogliani P, Matera MG. $\beta 2$-agonist therapy in lung disease. Am J Respir Crit Care Med 2013;187:690-6.

6. Matera MG, Page CP, Calzetta L, et al. Pharmacology and therapeutics of bronchodilators revisited. Pharmacol Rev 2020;72:218-52.

7. Cazzola M, Page C, Matera MG. Long-acting muscarinic receptor antagonists for the treatment of respiratory disease. Pulm Pharmacol Ther 2013;26:307-17.

8. Stolz D, Cazzola M. Characterising the cardiovascular safety profile of inhaled muscarinic receptor antagonists. In: Martínez-García MÁ, Pépin J-L, Cazzola M, editors. Cardiovascular complications of respiratory disorders (ERS Monograph). Sheffield, European Respiratory Society, 2020; p. $238-50$.

9. Cazzola M, Segreti A, Stirpe E, et al. Effect of an additional dose of indacaterol in COPD patients under regular treatment with indacaterol. Respir Med 2013;107:107-11.

10. Cazzola M, Brusasco V, Centanni S, et al. Project PriMo: sharing principles and practices of bronchodilator therapy monitoring in COPD: a consensus initiative for optimizing therapeutic appropriateness among Italian specialists. Pulm Pharmacol Ther 2013;26:218-28.

11. Calzetta L, Rogliani P, Matera MG, Cazzola M. A systematic review with meta-analysis of dual bronchodilation with LAMA/LABA for the treatment of stable COPD. Chest 2016;149:1181-96.

12. Calzetta L, Ora J, Cavalli F, et al. Impact of LABA/LAMA combination on exercise endurance and lung hyperinflation in COPD: A pair-wise and network meta-analysis. Respir Med 2017;129:189-98.

13. Calzetta L, Matera MG, Cazzola M. Pharmacological interaction between LABAs and LAMAs in the airways: optimizing synergy. Eur J Pharmacol 2015;761:168-73.

14. Calzetta L, Matera MG, Cazzola M. Pharmacological mechanisms leading to synergy in fixed-dose dual bronchodilator therapy. Curr Opin Pharmacol 2018;40:95-103.

15. Wedzicha JA, Decramer M, Ficker JH, et al. Analysis of chronic obstructive pulmonary disease exacerbations with the dual bronchodilator QVA149 compared with glycopyrronium and tiotropium (SPARK): a randomised, double-blind, parallelgroup study. Lancet Respir Med 2013;1:199-209.

16. Beeh KM, Burgel PR, Franssen FME, et al. How do dual longacting bronchodilators prevent exacerbations of chronic obstructive pulmonary disease? Am J Respir Crit Care Med 2017;196:139-49.

17. Cazzola M, Calzetta L, Puxeddu E, et al. Pharmacological characterisation of the interaction between glycopyrronium bromide and indacaterol fumarate in human isolated bronchi, small airways and bronchial epithelial cells. Respir Res 2016;17:70.

18. Yamada M, Ichinose M. The cholinergic pathways in inflammation: a potential pharmacotherapeutic target for COPD. Front Pharmacol 2018;9:1426.

19. Calzetta L, Matera MG, Rogliani P, Cazzola M. Dual 
LABA/LAMA bronchodilators in chronic obstructive pulmonary disease: why, when, and how. Expert Rev Respir Med 2018;12:261-4.

20. Cazzola M, Calzetta L, Ora J, et al. Searching for the synergistic effect between aclidinium and formoterol: From bench to bedside. Respir Med 2015;109:1305-11.

21. Cazzola M, Calzetta L, Segreti A, et al. Translational study searching for synergy between glycopyrronium and indacaterol. COPD 2015;12:175-81.

22. Cazzola M, Calzetta L, Page CP, et al. Pharmacological characterization of the interaction between aclidinium bromide and formoterol fumarate on human isolated bronchi. Eur J Pharmacol 2014;745:135-43.

23. Calzetta L, Rogliani P, Facciolo F, et al. Pharmacological characterization of the interaction between umeclidinium and vilanterol in human bronchi. Eur J Pharmacol 2017;812:147-54.

24. Calzetta L, Rogliani P, Page C, et al. Pharmacological characterization of the interaction between tiotropium bromide and olodaterol on human bronchi and small airways. Pulm Pharmacol Ther 2019;56:39-50.

25. Cazzola M, Matera MG. POINT: Should LAMA/LABA combination therapy be used as initial maintenance treatment for COPD? Yes. Chest 2018;154:746-8.

26. Cazzola M, Segreti A, Rogliani P. Comparative effectiveness of drugs for chronic obstructive pulmonary disease. Drugs Today (Barc) 2012;48:785-94.

27. Kerwin E, Ferguson GT, Sanjar S, et al. Dual bronchodilation with indacaterol maleate/glycopyrronium bromide compared with umeclidinium bromide/vilanterol in patients with moderate-to-severe COPD: results from two randomized, controlled, cross-over studies. Lung 2017;195:739-47.

28. Feldman GJ, Sousa AR, Lipson DA, et al. Comparative efficacy of once-daily umeclidinium/vilanterol and tiotropium/olodaterol therapy in symptomatic chronic obstructive pulmonary disease: a randomized study. Adv Ther 2017;34:2518-33.

29. Cazzola M, Rogliani P. Comparative effectiveness of indacaterol/glycopyrronium in the treatment of chronic obstructive pulmonary disease. J Comp Eff Res 2017;6:627-36.

30. Berenbaum MC. Isobolographic, algebraic, and search methods in the analysis of multiagent synergy. J Am Coll Toxicol 1988; 7:927-38

31. Calzetta L, Matera MG, Cazzola M, Rogliani P. Optimizing the development strategy of combination therapy in respiratory medicine: from isolated airways to patients. Adv Ther 2019;36:3291-8.

32. Rogliani P, Calzetta L, Braido F, et al. LABA/LAMA fixeddose combinations in patients with COPD: a systematic review. Int J Chron Obstruct Pulmon Dis 2018;13:3115-30.

33. Rogliani P, Matera MG, Ritondo BL, et al. Efficacy and cardiovascular safety profile of dual bronchodilation therapy in chronic obstructive pulmonary disease: A bidimensional comparative analysis across fixed-dose combinations. Pulm Pharmacol Ther 2019;59:101841.

34. Salanti G, Ades AE, Ioannidis JP. Graphical methods and numerical summaries for presenting results from multipletreatment meta-analysis: an overview and tutorial. J Clin Epidemiol 2011;64:163-71.

35. Calzetta L, Rogliani P, Page C, et al. Pharmacological characterization of the interaction between tiotropium bromide and olodaterol on human bronchi and small airways. Pulm Pharmacol Ther 2019;56:39-50.

36. Goldoni M, Johansson C. A mathematical approach to study combined effects of toxicants in vitro: evaluation of the Bliss independence criterion and the Loewe additivity model. Toxicol In Vitro 2007;21:759-69.

37. Benfante A, Braido F, Scichilone N. The anti-inflammatory properties of tiotropium. Lancet Respir Med 2018;6:e37.

38. Calverley PMA, Anzueto AR, Carter K, et al. Tiotropium and olodaterol in the prevention of chronic obstructive pulmonary disease exacerbations (DYNAGITO): a double-blind, randomised, parallel-group, active-controlled trial. Lancet Respir Med 2018;6:337-44.

39. Rogliani P, Calzetta L, Cazzola M. Is ICS-LAMA an alternative option to treat patients with COPD? Lancet Respir Med 2018;6:316-7.

40. Cazzola M, Rogliani P, Novelli L, Matera MG. Inhaled corticosteroids for chronic obstructive pulmonary disease. Expert Opin Pharmacother 2013;14:2489-99.

41. Anzalone G, Gagliardo R, Bucchieri F, et al. IL-17A induces chromatin remodeling promoting IL- 8 release in bronchial epithelial cells: effect of tiotropium. Life Sci 2016;152:107-16.

42. Profita M, Bonanno A, Montalbano AM, et al. $\beta 2$ long-acting and anticholinergic drugs control TGF- $\beta 1$-mediated neutrophilic inflammation in COPD. Biochim Biophys Acta 2012;1822:1079-89.

43. Wedzicha JA, Buhl R, Singh D, et al. Tiotropium/olodaterol decreases exacerbation rates compared with tiotropium in a range of patients with COPD: pooled analysis of the TONADO®/DYNAGITO ${ }^{\circledR}$ trials. Adv Ther 2020;37:4266-79.

44. Singh S, Loke YK, Enright PL, Furberg CD. Mortality associated with tiotropium mist inhaler in patients with chronic obstructive pulmonary disease: systematic review and metaanalysis of randomised controlled trials. BMJ 2011;342:d3215.

45. Rogliani P, Ritondo BL, Zerillo B, et al. Adding a second bronchodilator in COPD: A meta-analysis on the risk of specific cardiovascular serious adverse events of tiotropium/olodaterol fixed-dose combination. COPD 2020;17:215-23.

46. Maltais F, Buhl R, Koch A, et al. $\beta$-blockers in COPD: a cohort study from the TONADO research program. Chest 2018;153:1315-25.

47. Matera MG, Calzetta L, Cazzola M. $\beta$-Adrenoceptor modulation in chronic obstructive pulmonary disease: present and future perspectives. Drugs 2013;73:1653-63.

48. Cazzola M, Calzetta L, Rinaldi B, et al. Management of chronic obstructive pulmonary disease in patients with cardiovascular diseases. Drugs 2017;77:721-32.

49. Cazzola M, Matera MG. Combining dual bronchodilation and $\beta$-blockade in patients with an overlap between COPD and cardiovascular diseases. Chest 2018;153:1289-91.

50. Rinaldi B, Donniacuo M, Sodano L, et al. Effects of chronic treatment with the new ultra-long-acting $\beta 2$-adrenoceptor agonist indacaterol alone or in combination with the $\beta$-adrenoceptor blocker metoprolol on cardiac remodelling. Br J Pharmacol 2015;172:3627-37.

51. Miravitlles M, Baek S, Vithlani V, Lad R. Optimal bronchodilation for COPD patients: are all long-acting $\beta 2$-agonist/longacting muscarinic antagonists the same? Tuberc Respir Dis (Seoul) 2018;81:198-215. 